\title{
A COMPARISON OF THE FATIGUE BEHAVIOR OF HUMAN TRABECULAR AND CORTICAL BONE TISSUE
}

\author{
K. ChOI and S. A. GoldsteIN* \\ Orthopaedic Research Laboratories, The University of Michigan, Section of Orthopaedics, Department \\ of Surgery and the Bioengineering Program, Ann Arbor, Michigan, U.S.A.
}

\begin{abstract}
The fatigue properties of trabecular bone tissue (single trabeculae) and similarly sized cortical bone specimens from human tibia were experimentally determined on a microstructural level using fourpoint bending cyclic tests, and they were compared based on modulus, mineral density, and microstructural characteristics. The results showed that trabecular specimens had significantly lower moduli and lower fatigue strength than cortical specimens, despite their higher mineral density values. Fracture surface and microdamage analyses illustrated different fracture and damage patterns between trabecular and cortical bone tissue, depending upon their microstructural characteristics. Based on the results from mechanical tests and qualitative observations, a possible mechanical role of the cement lines in trabecular tissue microfracture was suggested.
\end{abstract}

\section{INTRODUCTION}

Fatigue failures in bone have been observed clinically after prolonged or rigorous exercise (Bargren and Tilson. 1971; Devas, 1958; McBryde, 1976). Femoral neck fractures of elderly persons might also be related to fatigue failure (Freeman et al., 1974; Griffiths et al., 1971; Watson, 1975). Although the fatigue failure mechanism in bone is not completely understood, the fatigue behavior of bone resembles composite materials, exhibiting a gradual loss of stiffness and strength throughout cyclic loading due to fatigue damage accumulation (Carter and Hayes, 1977; Keller et al., 1985; Schaffler et al., 1989; Pattin et al., 1990). Damage has been observed in bone specimens subjected to repetitive loading even within normal physiological stress or strain ranges (Frost, 1960; Freeman et al., 1974; Schaffler et al., 1989). It has also been hypothesized that the fatigue damage accumulation might initiate or control bone remodeling (Currey, 1962; Frost, 1966; Baker et al., 1972; Radin, 1972; Martin and Burr, 1982; Burr et al. 1985; Carter and Caler, 1985). Due to the implications on clinical problems and remodeling mechanisms, the fatigue behavior of bone has been extensively investigated. The fatigue strength of bone has been reported to be a function of the loading mechanism, frequency (Lafferty, 1978: Lafferty and Raju, 1979), strain rate (Schaffler et al., 1989), age (Swanson et al., 1971; Keller et al., 1985), anatomic site (Evans and Lebow, 1957), stiffness (Carter and Hyes, 1977; Carter et al., 1981), density (Carter and Hayes, 1976; Carter et al., 1976) and temperature (Carter and Hayes, 1976), as well as microstructure (Carter et al. 1976; Evans and Riolo, 1970; Moyle et al., 1978; Moyle and Bowden, 1984; Corondan and Haworth, 1986).

Received in final form 9 June 1992

*Author to whom correspondence should be addressed at: Orthopaedic Research laboratories, G-0161 400 N. Ingalls, Ann Arbor. MI 48109-0486. U.S.A.
The studies described above have focused on cortical bone. The fatigue behavior of trabecular bone tissue (single trabeculae) is virtually uncharacterized, except for a preliminary study from our laboratory (Choi and Goldstein, 1989). Determining the fatigue properties of trabecular bone tissue is important to better understand its normal mechanical and physiologic behavior as well as its response to orthopedic procedures on a microstructural level. These include fatigue fractures of trabecular bone, bone-implant interface mechanics (Pedersen et al., 1990), and remodeling mechanisms (Hollister et al., 1990).

Trabecular bone tissue has a microstructure different from cortical bone. It is composed of thin plateshaped structural units, called 'trabecular packets'. and it has a 'mosaic-like' microstructure (Jee, 1983; Kragstrup and Melsen, 1983; Parfitt, 1983). Several cement lines with random orientation exist in trabecular tissue. The effects of the microstructure of trabecular tissue on elastic modulus have been previously investigated and reported to be significant by the authors (Choi et al., 1990). Thus, it may be hypothesized that the unique microstructure of trabecular tissue results in fatigue behavior different from cortical bone tissue.

The purpose of this study was to report initial fatigue data obtained from human trabecular and cortical bone tissue using four-point cyclic tests, to compare them on the basis of microstructure, mineral density and modulus and, hopefully, to provide some insight into the understanding of bone micro-mech anics under repetitive loading.

\section{MATERIALS AND METHODS}

All specimens were obtained from a pair of human tibia (59 years old, healthy male). While trabecular specimens were machined from the proximal tibia (top $8 \mathrm{~mm}$ ), cortical specimens were obtained from the 
tibial diaphyses. The tibiae were obtained within $72 \mathrm{~h}$ of death and stored at $-30^{\circ} \mathrm{C}$ until machining.

\section{Specimen preparation}

Regularly shaped rectangular beam specimens were produced using procedures (Fig. 1) previously described by the authors (Choi et al., 1990). A series of thin bone sections were cut on a low-speed diamondblade saw (Model 11-1180 Isomet, Buehler, Lake Bluff, IL) followed by milling using a specially designed milling machine with a miniature drill bit of $200 \mu \mathrm{m}$ diameter (sectioning-milling). Trabecular specimens were machined from both vertically and horizontally oriented single trabeculae, and cortical specimens were oriented parallel to the long axis of the tibia. An additional set of cortical specimens was prepared using only the low-speed saw (sectioning only) in order to evaluate the effects of machining methods on the fatigue properties.

Specimen dimensions were measured by using a digital image processing system (Recognition Technology Inc., Westborough, MA) hosted by an IBM microcomputer. Each specimen was observed through a light microscope with $\times 25$ objective lens, and imaged with a video camera (Series 68, Dage-MTI Inc., Michigan City, IN). The dimensions were determined by digitizing the image at a magnification of $0.95 \mu \mathrm{m} \mathrm{pxl}^{-1}$. Both specimen height and base ranged from 70 to $140 \mu \mathrm{m}$. Only specimens with base-toheight ratios between 0.5 and 2.0 were included in this study. Specimens were kept moist during the procedure and stored at $-30^{\circ} \mathrm{C}$ until testing.

A total of 23 trabecular and 31 cortical specimens were prepared and separated into three testing groups based on bone types and preparation methods (Table 1). Cortical specimens in Group 2 were prepared to compare the fatigue properties between cortical and trabecular tissue (Group 1) under the same conditions. Cortical specimens in Group 3, machined by the sectioning-only procedure, were prepared as a control group to evaluate the effects of surface finish on the fatigue properties.

\section{Mechanical test}

The fatigue testing apparatus, shown in Fig. 2, was developed to load specimens in cyclic four-point bending. A platform, providing end supports for the specimens and an irrigation chamber, was placed in series with a high-resolution load cell (Model FTD-G100, Schaevitz Engineering, Camden, NJ), and attached to a stationary base. The loading head was integrated into a specially designed electromagnetic actuator, which provided the loading mechanism. In order to provide even load distribution through two knife edges, a bearing rod was placed between the load cell and the platform (Fig. 3). The knife edges were rounded at the ends to minimize stress concentrations around contact areas. A servovalve control system (Model ECM 2-L1-D, Continental Hydraulics, Savage, MN) piloted by a signal generator (Model 3300A Hewlett Packard, Loveland, CO) provided the desired control parameters to the actuator. In order to maintain a desired load level on the specimens, the output signal from the load cell was directed to the control system through a feedback mechanism.

Prior to fatigue testing, static 'pre-yield' four-point bending tests were conducted on both trabecular and cortical specimens. Each specimen was loaded to

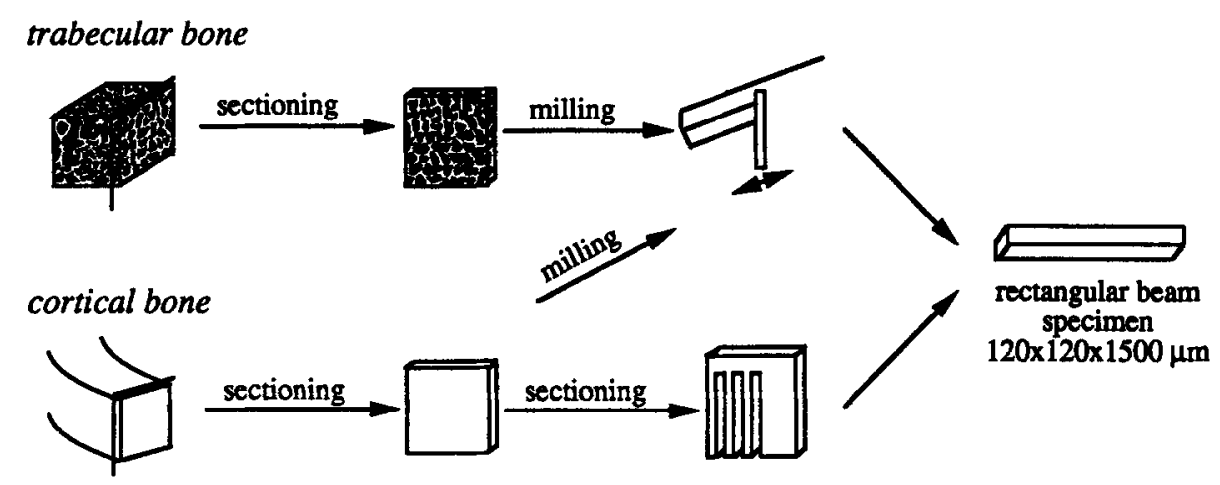

Fig. 1. Schematic diagram of specimen preparation procedures (sectioning-milling and sectioning only).

Table 1. The specimen groups based on bone type and preparation technique

\begin{tabular}{lllccc}
\hline Bone type & $\begin{array}{c}\text { Preparation } \\
\text { technique }\end{array}$ & $n$ & $\begin{array}{c}\text { Maximum stress } \\
\text { range (MPa) }\end{array}$ & $\begin{array}{c}\text { Number of } \\
\text { stress levels }\end{array}$ \\
\hline Group 1 & Trabecular & Sectioning-milling & 23 & $100-140$ & 5 \\
Group 2 & Cortical & Sectioning-milling & 16 & $150-180$ & 4 \\
Group 3 & Cortical & Sectioning only & 15 & $150-180$ & 4 \\
\hline
\end{tabular}




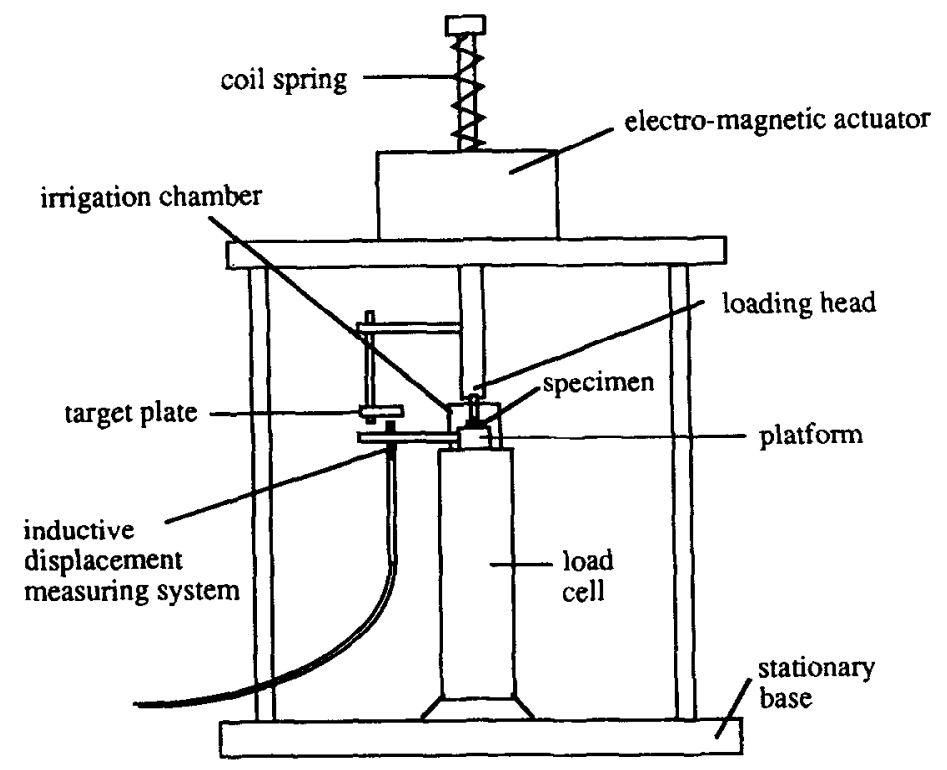

Fig. 2. Schematic diagram of the four-point bending test apparatus.

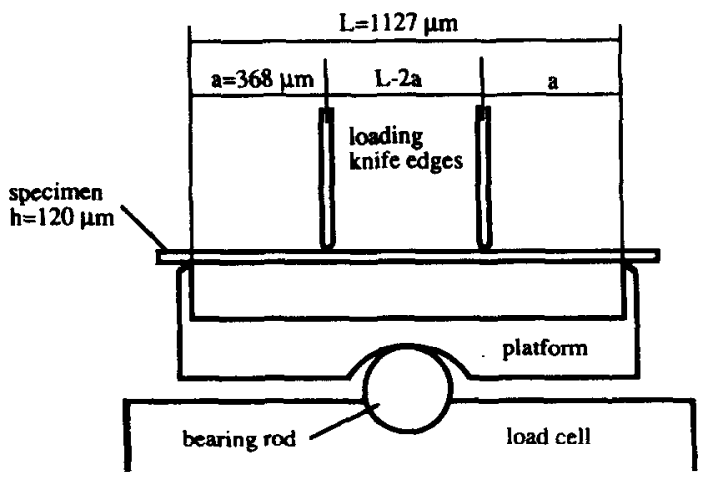

Fig. 3. Schematic diagram of the four-point bending mechanism.

approximately $0.05 \mathrm{~N}(\sim 25 \%$ of the average failure load), avoiding permanent damage to the specimen. A high-resolution inductive displacement measuring system (KD-2300-.5SU, Kaman Instrumentation Co., Colorado Springs, $\mathrm{CO}$ ), placed between the load cell and loading head, was used to measure the deflection of the specimen. Each specimen was loaded at a constant loading rate of $0.02 \mathrm{Ns}^{-1}$. From the load-deflection data, the elastic modulus of each specimen was calculated using linear elastic beam theory. The dimensions of span length $(L)$ and the distance between a knife edge and support in the apparatus are illustrated in Fig. 3. Since the average specimen height $(h)$ was approximately $120 \mu \mathrm{m}$, the aspect ratio $\left(L h^{-1}\right)$ was approximately 10 .

After static testing, the specimens were tested in cyclic four-point bending. The displacement measuring device was not used during fatigue testing

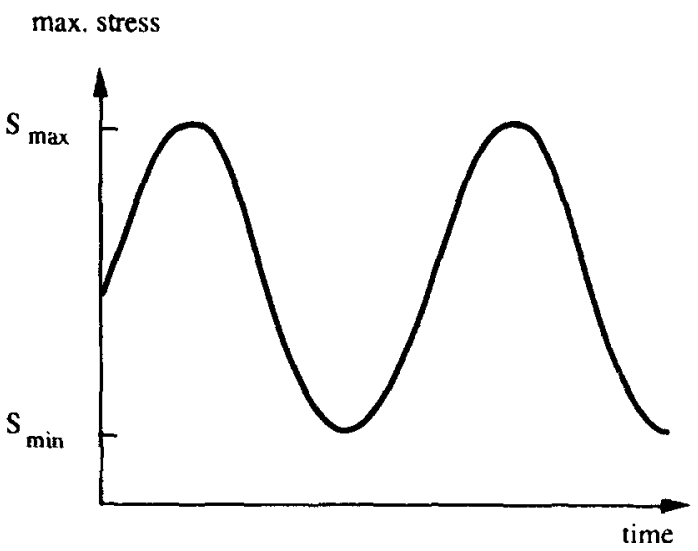

Fig. 4. A pulsating sinusoidal waveform. While the max imum stress values varied, the minimum stress was fixed at approximately $20 \mathrm{MPa}$. Testing was performed at a frequency of $2 \mathrm{~Hz}$, in water at room temperature.

in order to protect the transducer from crushing when failure occurs. The type of stress variation in the cyclic bending was a pulsating sinusoidal loading (Fig. 4). Each specimen was tested at a frequency of $2 \mathrm{~Hz}$, in water at room temperature, until complete failure. The test was discontinued after 200,000 cycles if no failure occured. While the minimum bending stress $\left(S_{\min }\right)$ was fixed at approximately $20 \mathrm{MPa}$ for all the specimens, the maximum stress values $\left(S_{\max }\right)$ varied, depending upon the stress level applied and the specimen groups (Table 1). The maximum and minimum stress values in this study represent the outer fiber tensile stresses of the beam specimens, and they were calculated using 
the following equation:

$$
\mathrm{S}=\frac{(P / 2) a(h / 2)}{I},
$$

where

$$
\begin{aligned}
& S=\text { outer fiber tensile stress }(\mathrm{MPa}), \\
& P=\text { applied load }(\mathrm{N}), \\
& a=\text { distance between a knife edge and support }(\mathrm{mm}), \\
& I=\text { area moment of inertia }\left[=(1 / 12) b h^{3}\right]\left(\mathrm{mm}^{4}\right) . \\
& h=\text { specimen height }(\mathrm{mm}), \\
& b=\text { specimen base }(\mathrm{mm}) .
\end{aligned}
$$

\section{Measurements of mineralization}

An automated microradiography technique (microcomputed tomography) was used to determine relative differences in mineral density between specimens. The application of this technique to microspecimens of bone has previously been described in detail (Kuhn et al., 1989; Choi et al., 1990). Twenty-three trabecular specimens from Group 1 and 15 cortical specimens from Group 2 were mounted on a platform and scanned sequentially at a high magnification

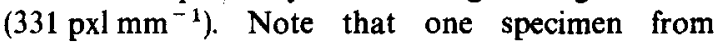
Group 2 was lost during this procedure. The relative mineral density (unit, $U=$ grey scale $\mathrm{mm}^{-2}$ ) was determined by integrating each X-ray attenuation profile, assumed to be proportional to mineral density, and normalizing by the specimen cross-sectional area. This measure represents relative differences in mineral density between specimens, which are sufficient to investigate the effects of mineral density on modulus and fatigue properties.

\section{Fracture surfaces and microstructure}

Scanning electron microscopy (SEM) along with backscattered image (BSI) analyses were conducted to observe the fracture patterns and microdamage of fatigued specimens. Each specimen was coated with gold-platinum and scanned at approximately $\times 400$ magnification (Autoscan Model U-2, ETEC Co., Hayward, CA) to observe the fracture patterns. Then, the specimens were embedded in Koldmount self-curing resin (Vernon-Benshoff Co., Albany, NY), polished, carbon-coated, and scanned to identify microcracks and structural variations.

\section{Statistical analysis}

Two-tailed Student's $t$-tests were performed to compare modulus and mineral density values and analysis of covariance was used for a comparison of slopes and intercepts in $\mathrm{S}-\mathrm{N}$ curves between the specimen groups. The significance was determined at a $p$ value of 0.05 .

\section{RESULTS}

Cortical bone specimens had a significantly higher modulus $(18 \%)$ than trabecular specimens $(p<0.01)$. The average mineral density of cortical specimens is, however, $6 \%$ lower than that of trabecular specimens $(p=0.05$, Table 2). No significant difference in modulus was found between cortical specimen Group 2 (sectioning milling) and Group 3 (sectioning only).

Fatigue results are summarized in the form of median $S-N$ curves (Fig. 5). The numbers on the arrows indicate the number of specimens not failed after 200,000 cycles (run-out specimens). The $S-N$ relationships can be expressed by the following linearized equation:

$$
\log N=A+B S
$$

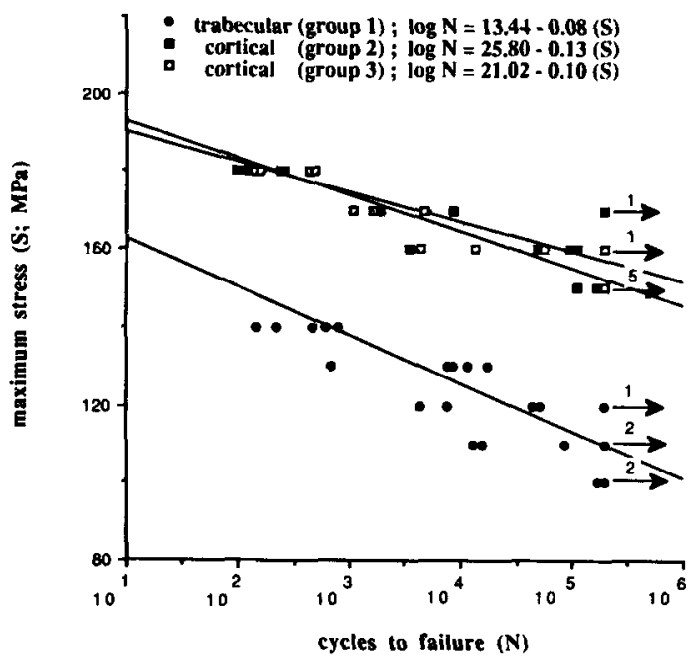

Fig. 5. Median $S-N$ curves for each specimen group. The numbers on arrows indicate the number of run-out specimens for given stress levels.

Table 2. The modulus and mineral density measurements results for specimen Groups 1, 2, and 3 . Modulus values are reported in units of $\mathrm{GPa}$, and mineral density measures in units of $U$ (grey scale $\mathrm{mm}^{-2}$ ). Standard deviations are shown in parentheses. $p$-values from two-tailed Student's $t$-tests indicate the significance of the differences between groups

\begin{tabular}{lcccc}
\hline & Modulus & Significance & Mineral density & Significance \\
\hline Trabecular bone & $5.72(1.27)$ & & $0.294(0.030)$ & \\
(Group 1) & $n=23$ & $p<0.01$ & $n=23$ & $p=0.05$ \\
Cortical bone & $6.75(1.00)$ & & $0.277(0.016)$ & \\
(Group 2) & $n=16$ & Not significant & $n=15$ & \\
Cortical bone & $6.48(1.61)$ & & Not available & \\
(Group 3) & $n=15$ & & \\
\hline
\end{tabular}




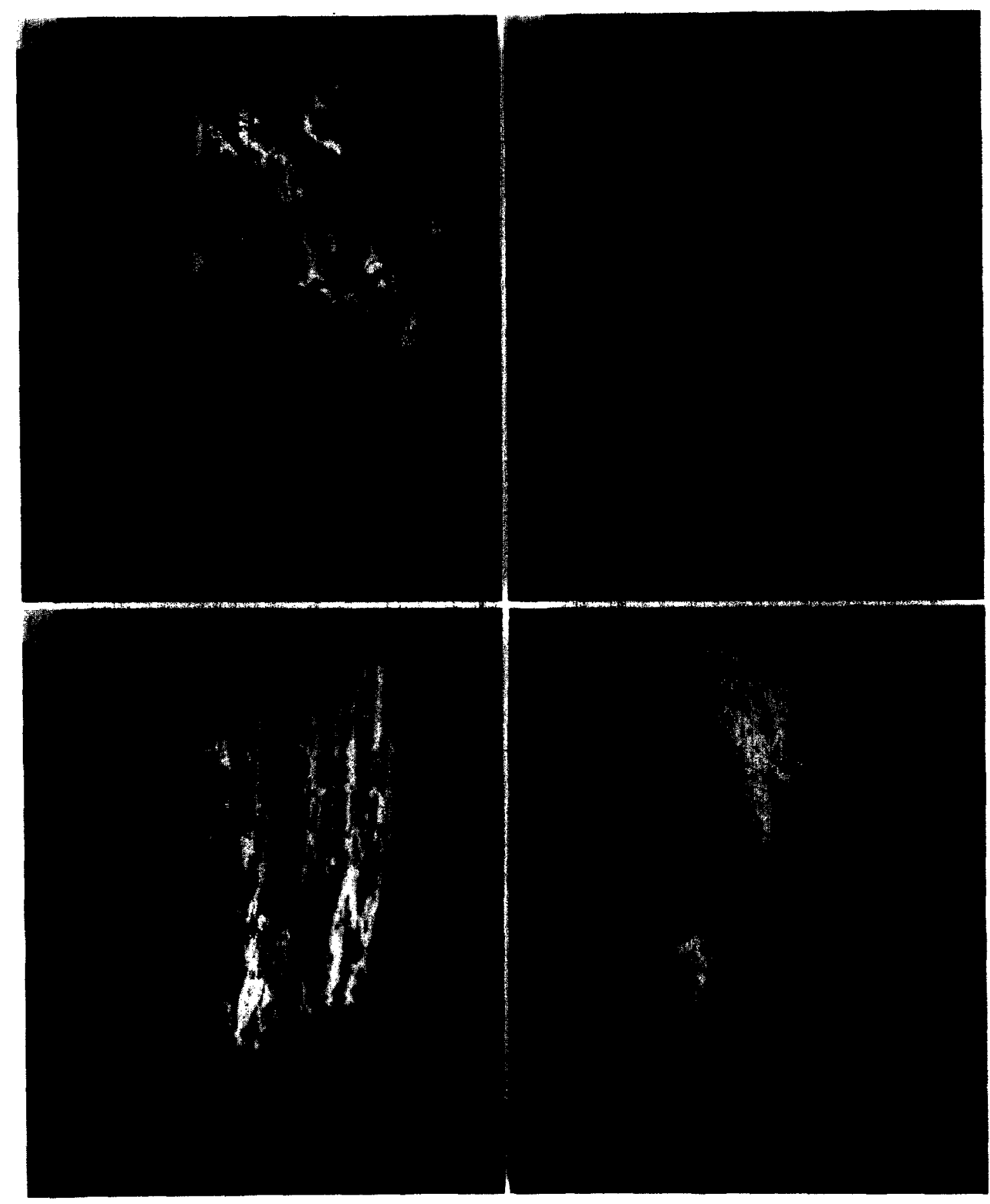

Fig, 6. SEM analyses $(\times 400)$ on fracture surfaces. The surfaces of cortical specimens $(a, b)$ reveal peak-and-valley-type fracture (indicated by arrows) and layers of lamellae structure with rough surfaces, but the surfaces of trabecular specimens show oblique (c) and haphazard (d) fracture patterns with relatively smooth surfaces. 

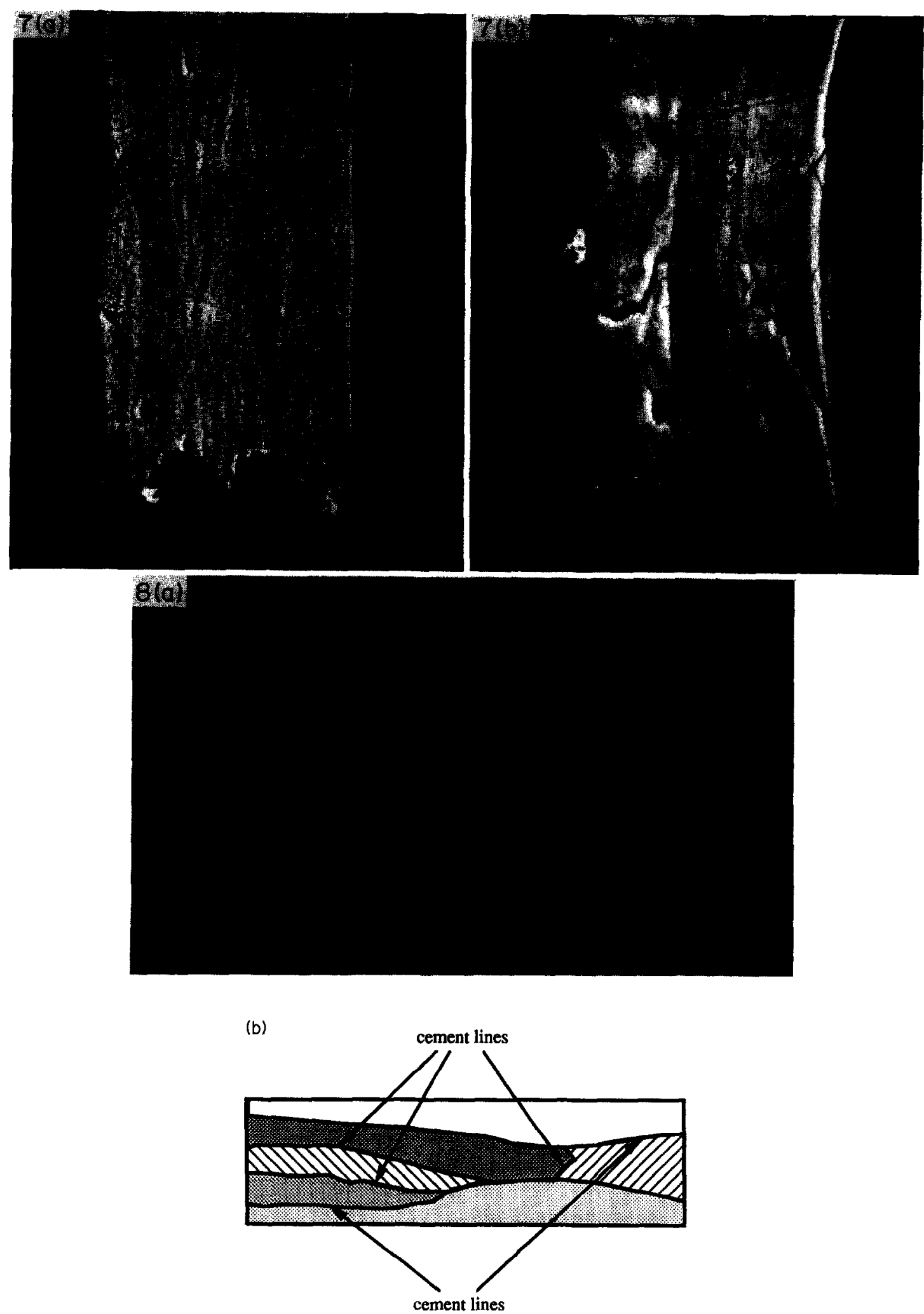

Fig. 7. BSI analyses $(\times 400)$ on cortical (a) and trabecular (b) specimens. Cortical specimens show zigzag crack pathway, indicated by arrows. In trabecular specimens, several microcracks (indicated by arrows) associated with cement lines were observed. Thin straight lines found in both picture represent artifacts due to surface polishing.

Fig. 8. The 'mosaic-like' structure of trabecular bone tissue: (a) a backscattered image $(\times 400)$ and $(b)$ its simplified schematic diagram, which illustrate several trabecular packets (about six) and cement lines. 
where $A$ and $B$ are constants, and $S$ refers to the maximum stress value. The regression coefficients $A$ and $B$ in equation (2) for each specimen group were noted in Fig. 5. Fatigue strength is a stress value for failure at a given number of cycles, as determined from an $S-N$ curve.

No significant difference in fatigue strength was found between cortical specimen Group 2 (sectioning-milling) and Group 3 (sectioning only). Cortical specimens showed higher fatigue strength than trabecular specimens. Most of the cortical specimens underwent relatively small deformation, and all cortical specimens were fractured completely. By contrast, trabecular specimens showed a large deformation before failure, and some trabecular specimens were significantly deformed without complete fracture. All the fractures occured either between the two loading kinfe edges or across the knife edges. The specimens fractured clearly outside the edges were not included in this study.

Run-out trabecular specimens $(n=5)$ did not demonstrate large deformations, and they had slightly higher average moduli $(6.25 \pm 0.99 \mathrm{GPa})$ and mineral densities $(0.303 \pm 0.020 \mathrm{U})$ than the other trabecular specimens $(5.57 \pm 1.32 \mathrm{GPa}, p=0.29 ; 0.292 \pm 0.032 \mathrm{U}$. $p=0.47$ ). Run-out cortical specimens (Group 2, $n=3$ ) also had slightly higher moduli $(7.01 \pm 0.10 \mathrm{GPa})$ and mineral densities $(0.288 \pm 0.024 U)$ than the other cortical specimens $(6.68 \pm 1.03 \mathrm{GPa}, \quad p=0.62 ; \quad 0.275$ $\pm 0.015 \mathrm{U}, p=0.31$ ).

Different fracture patterns were observed from each bone type [Fig. 6(a)-(d)]. In cortical bone, transverse fractures, where the fracture surfaces are perpendicular to the long axis of specimens, were observed in most of the specimens. Delamination was often observed on the tensile sides of some specimens. Fracture surfaces of cortical bone exhibited layers of plateshaped lamellar structure and showed a peak-andvalley-type fracture pattern. A zigzag crack pathway observed in cortical specimens [Fig .7(a)] also indicated the peak-and-valley fracture pattern. On the other hand, in trabecular specimens, oblique fracture with an angle of approximately $45^{\circ}$ was a typical pattern [Fig. 6(c)]. Fracture of the plate-shaped lamellae observed in cortical specimens was not found in the fracture surfaces of trabecular specimens, and the texture of the surfaces looked relatively smoother. More complicated fracture patterns were also observed in trabecular specimens [Fig. 6(d)], which might reflect the complex nature of their microstructure. Multiple fractures were often observed in trabecular specimens. SEM analyses revealed no crushing or permanent marks around the contact areas between the knife edges and specimen surfaces.

Different microstructure and fatigue damage patterns between trabecular and cortical tissue were observed in BSI images [Fig. 7(a) and (b)]. Cortical specimens demonstrated a well-organized structure with uniform mineral distribution and almost no cement lines were observed due to their subosteonal specimen size. Almost no microcracks were found in cortical specimens. By contrast, trabecular specimens illustrated 'mosaic-like' structures with uneven mineral distribution within a specimen, and several cement lines and packets were observed [Fig. 8(a) and (b)]. Several microcracks, mostly associated with cement lines or lacunae, were found throughout the trabecular specimens [Fig. 7(b)].

\section{DISCUSSION}

In this investigation, four-point bending fatigue tests were conducted on the microbeam specimens from human single trabeculae and cortical bone. The effects of differences in microstructure, mineral density and modulus between the two bone tissues on the fatigue behavior have been examined. While the bending test is a standard testing method for normal engineering materials, it raises several concerns when it is used for composite materials like bone. There also exist some concerns about specimen preparation methods in this study.

The possible artifactual defects on the specimens due to machining have been previously examined by the authors utilizing both histological and BSI analyses (Choi et al., 1990), where no artifactual defects were observed under high magnification. Swanson et al. (1971) showed that the effects of surface finishes on fatigue properties were not significant. They also concluded that biological defects such as lacunae or canaliculi exert a greater effect on fatigue properties than the defects caused by the machining process. In the present study, this aspect was further evaluated by fatigue testing cortical specimens prepared by two different machining processes (sectioning-milling versus sectioning only), since specimen size in this study was much smaller than that in Swanson's study. $S-N$ curves from the two cortical specimen groups ( 2 and 3 ) showed no significant differences (Fig. 5), which suggests that the surface effects produced by sectioning-milling are comparable to those by sectioning only. and that the milling technique is a viable method to prepare microspecimens of cortical bone for fatigue tests. However, it is not clear that this is true for trabecular tissue since some interactions could be produced between milling and microstructure of trabecular tissue.

One of the concerns about the testing methodology was that a highly uneven contact between loading knife edges and specimen could introduce a significant error. This aspect was evaluated by comparing fatigue data from our previous three-point bending study (Choi and Goldstein, 1989) with those from this study. No significant differences in either modulus or fatigue properties were found between the two bending methods (Fig. 9). This result, along with an inspection of the fracture sites, which were observed to occur between the loading knife edges in most of the specimens, suggest that this is not a significant source of error in our experiments. 


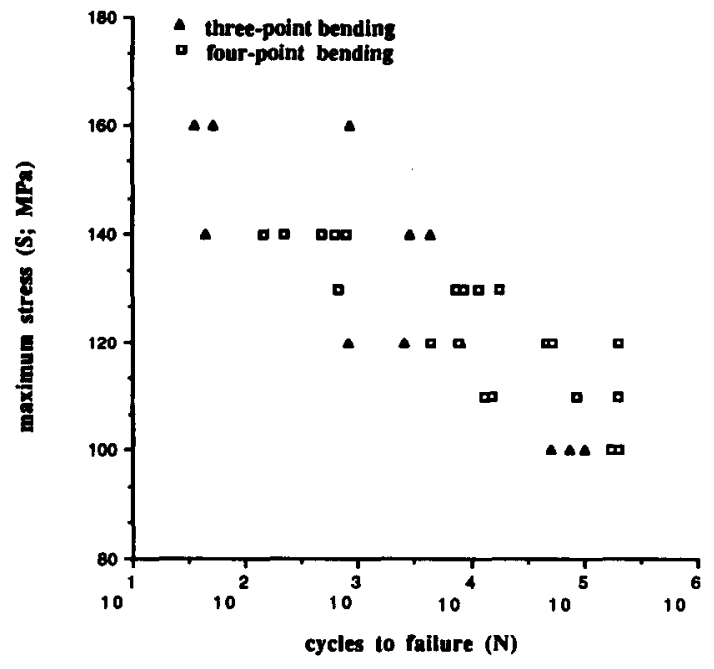

Fig. 9. Comparison of fatigue data from the current study (four-point bending) with those from our previous study (three-point bending), indicating no significant difference.

Bending tests on bone material, however, introduce several concerns, including the effects of anisotrophy and heterogeneity on stress distributions (Tarnopol'skii and Kinics, 1985) and the effects of yielding due to the concentrated loading on stress estimations (Burstein et al., 1972). While it is very difficult to accurately estimate the total error in stress estimation due to anisotropy and heterogeneity, Martin and Pramanik (1989) recently suggested that bone specimens with an aspect ratio greater than 10 seem to have less effects than short, thick specimens with a ratio of less than four. Burstein et al., (1972) reported that the effects of yielding due to concentrated loading in bending could introduce a significant overestimation of ultimate stress in large cortical specimens. The stress levels used in this study did not produce apparent permanent crushing around loading points, perhaps due to a significantly small loading over contact lines $(0.05-0.1 \mathrm{~N}$ over $0.12 \mathrm{~mm})$ compared to those used in whole bone bending tests $2000-6000 \mathrm{~N}$ over a few $\mathrm{mm}$, resulting in more than 500 times higher contact stresses), where crushings were found (Torzilli et al., 1981; Martens et al., 1986). It is not clear, however, how much yielding and overestimation could have been introduced in microspecimens at the stress levels. It should be noted that the yeild stress of trabecluar tissue may be different from that of cortical tissue; thus, one may expect different effects of yielding between the two tissues even under the same stress level.

The fatigue strength of cortical specimens in this study was compared with that in other studies in Fig. 10 (Carter et al., 1976, 1981; Carter and Hayes, 1976, 1977; Gray and Korbacher, 1974; Swanson et al., 1971; Lafferty and Raju, 1979). The differences in testing methods, testing conditions, and materials may

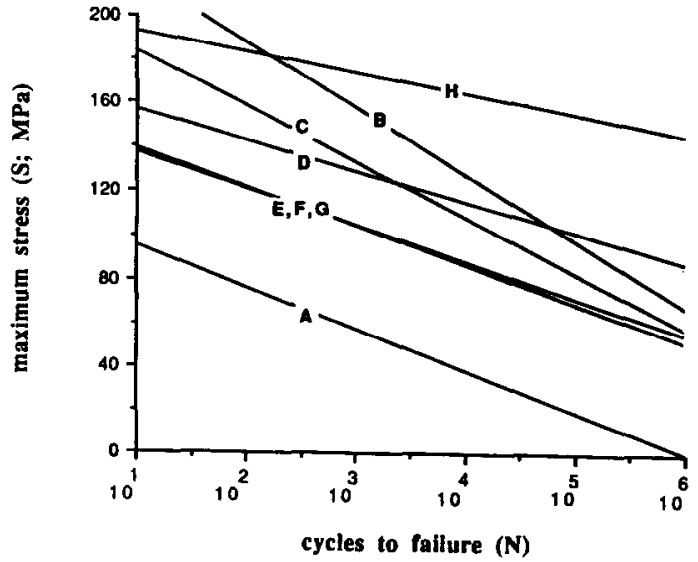

Fig. 10 Comparison of fatigue strength of cortical bone from the current study with those from other studies. Note that all the data from the other studies were transformed into semilog coordinates and plotted through linear regressions. (a) Carter et al. (1981): human, uniaxial, $37^{\circ} \mathrm{C}, 0.5-1 \mathrm{~Hz}$; (b) Carter and Hayes (1976): bovine, rotating bending, $21^{\circ} \mathrm{C}$, $125 \mathrm{~Hz}$; (c) Carter and Hayes (1976): bovine, rotating bending, $45^{\circ} \mathrm{C}, 125 \mathrm{~Hz}$; (d) Gray and Korbacher (1974): bovine, compressive, $22^{\circ} \mathrm{C}, 30 \mathrm{~Hz}$; (e) Swanson et al. (1971): human, rotating bending, room temperature, $70 \mathrm{~Hz}$; (f) King and Evans (1967): human (embalmed), flexural, room temperature, $30 \mathrm{~Hz}$; (g) Lafferty and Raju (1979): bovine, rotating bending, $21^{\circ} \mathrm{C}, 30 \mathrm{~Hz}$; (h) Current study: human, four-point bending, room temperature, $2 \mathrm{~Hz}$.

explain the variations in fatigue strength among the studies. The anisotropic nature of bone material makes bone behave differently under different loading regimes. Cortical bone exhibited higher fatigue strength under compressive loading (Gray and Korbacher, 1974) than under either fully reversed rotating bending (Swanson et al, 1971; Carter and Hayes, 1977; Carter et al., 1976) or uniaxial tensile-compressive loading (Carter et al., 1981). The effect of a range of stress less than complete reversal on the fatigue strength for metal specimens has been well documented, and the general nature of this effect is that the lower range of stress found in pulsating loading, compared to a fully reversed protocol, results in the higher fatigue strength. A pulsating loading was used in this study. The effects of temperature may be another factor which needs to be considered. Carter $e t$ al. (1976) found that decreasing the temperature from 37 to $21^{\circ} \mathrm{C}$ would increase the fatigue life of bovine cortical bone by more than $200 \%$. Temperature was maintained at $21^{\circ} \mathrm{C}$ in this study. Lafferty (1978) reported that loading frequencies less than $30 \mathrm{~Hz}$ would not affect the fatigue life; however, frequencies higher than $30 \mathrm{~Hz}$ increase the fatigue life. A frequency of $2 \mathrm{~Hz}$ was used in this study. Another factor that may contribute to the measured properties is specimen size, since the heterogeneous and complex nature of bone material provides different structures at every level of organization. The effects of specimen size have been emphasized by several investigators (Reilly and Burstein, 1974; Rice et al., 1988; Kuhn et al., 1989) and 
they proved to be significant in the static modulus measurements (Choi et al., 1990). However, the effect of specimen size on the fatigue behavior is unclear. It should be noted that since many factors affect the fatigue behavior of bone, valid comparisons of the fatigue data can only be made if all the factors are the same between studies.

The specimens in this study were obtained from only one cadaver and from a very localized anatomic site in order to eliminate the effects of donor and anatomic regions. Therefore, the results in this study may not represent the overall fatigue properties of bone tissue. We believe, however, that the results would still provide some insight into the understanding of bone micromechanics under repetitive loading.

Fatigue results showed significantly lower fatigue strength in trabecular specimens than in cortical specimens. The modulus and mineral density results demonstrated that trabecular specimens had a lower modulus than cortical specimens, while trabecular specimens had higher mineral density (Table 2), which agrees well with our previous findings (Choi et al., 1990). While both modulus and fatigue strength of bone are heavily dependent on its structure (Martin and Ishida, 1989; Schaffler and Burr, 1988; Wright and Hayes, 1976; Choi et al., 1990), mineral density does not provide any information about the structurc. Mineral density information alone might not be a sufficient estimator for modulus or fatigue strength unless microstructural variations are accounted for, when structurally different bones are being compared. Carter et al. (1981) showed that modulus was the most effective variable to explain their fatigue data among porosity and density variables. Carter et al. (1976) also investigated the effects of microstructure on the fatigue life of bovine cortical bone. According to their regression model, when fully primary bone and fully secondary bone were compared, the effects due to microstructural changes on fatigue strength were about five times greater than those due to density changes.

The significant difference in the fatigue properties between trabecular and cortical specimens in this study might have resulted from the differences in their microstructure along with the loading mechanism (Fig. 11). Cortical specimens consist of layers of plateshaped lamellae with none or one cement line, since the specimens are smaller than a single osteon. The orientation of both the lamallae and the cement line is parallel to the long axis of the specimen, but perpendicular to the loading direction [Fig. 11(a)]. In cortical specimens, it is likely that cracks would be initiated at biological defects on the surfaces and propagated through the layers of lamellae. At a microscopic level, crack propagation seems to correlate with lamellar structure. While it is not clear whether lamellae consist of alternating fibrous and cementing layers (dense and loose lamellae: Marotti, 1988) or fibrous layers in which the collagen fiber orientation changes from one layer to a successive layer (Gebhardt's model; Ascenzi

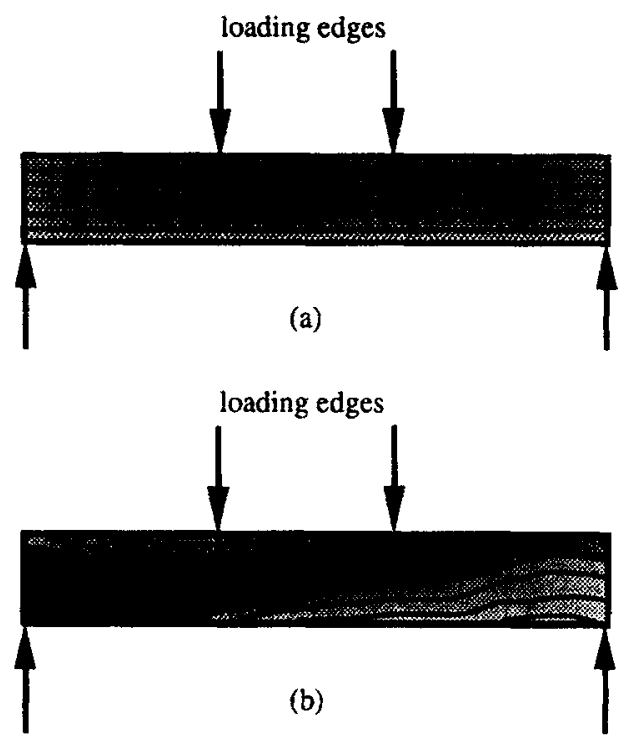

Fig. 11. A simplified diagram showing the microstructural characteristics of cortical (a) and trabecular (b) specimens. Note the relative lamellar orientation with respect to loading direction. The degree of mineralization is illustrated by different grey levels.

and Bonucci, 1976), the structural changes between lamellae might have resulted in the changes in crack direction, thus producing a zigzag crack pathway [Fig. 7(a)] and transverse fractures with peak-andvalley surfaces [Fig. 6(a) and (b)]. These rougher fracture surfaces indicate more energy dissipation for creating surfaces, slower crack propagation and higher fatigue strength in cortical specimens.

Unlike cortical bone osteons, trabecular tissue consists of trabecular packets, due to its remodeling characteristics. These packets are generally thin and plate-shaped and delineated by several cement lines, producing 'mosaic-like' microstructure (Fig. 8; Kragstrup and Melsen, 1983; Jee, 1983; Parfitt, 1983). Schaffler et al., (1987) reported that the cement line in human cortical bone has both lower calcium and phosphate concentrations than adjacent bone, thus providing a compliant interface. As illustrated in Fig. 11(b), in trabeculae under bending load, cracks would be initiated at the intersections of cement lines with the stressed surfaces and/or biological defects and propagated through cement lines, where higher shear stresses may exist due to the possible stiffness difference between bone and cement lines (Burr et al., 1988). While we do not expect crack propagation only along cement lines, since cement lines do not always go completely across trabeculae, we think that several initial cracks and their partial propagation along cement lines, particularly around the stressed surfaces, would result in a significant loss of structural rigidity in such small specimens. Therefore, complete fractures would occur in a relatively short time period after the initial cracking. Several microcracks observed along 
cement lines [Fig. 7(b)] suggest that continuous loading will fuse the cracks and eventually lead to complete failure. This multiple-crack initiation and growth might be responsible for the large deformation before failure and multiple fractures often observed in trabecular specimens. Fractures through the complaint cement lines might also result in smoother fracture surfaces and the oblique and haphazard fracture patterns [Fig. 6(c) and (d)], which likely reflect the geometry or distribution pattern of cement lines within trabeculae. The smoother fracture surfaces indicate less energy dissipation, fastcr crack propagation and lower fatigue strength.

The mechanical role of cement lines in cortical bone has been suggested by Burr et al.,(1988). The cement lines in cortical bone may arrest or slow down crack propagation by providing significant local stiffness changes (Kendall, 1975), thus increasing the fatigue strength (Frost, 1986). Accordingly, cortical bone with higher osteonal density (more cement lines) would have better fatigue resistance by providing more local interfaces, that is, providing a 'bone-cementline-bone-cement-line ...' crack propagation pathway (Evans and Riolo, 1970; Moyle et al., 1978; Moyle and Bowden, 1984; Corondan and Haworth, 1986). Although we may assume the same compositional and mechanical properties of cement line for both cortical and trabecular tissue, the crack propagation mechanism for large cortical bone specimens may not occur in small trabeculae, mainly due to the different geometry and density of cement lines and the different structural level (macro versus micro) between the two tissues. In trabecular bone tissue, cracks would follow the 'bone-cement-lines-fracture' pathway instead of the 'bone-cement-line-bone-cement-line...' pathway.

Acknowledgements - Support for this study was provided by a grant from the National Institute of Health (AR34399, AR31793), the Whitaker Foundation, and an OREF-Brystol Meyers/Squibb/Zimmer award. The authors thank Dr J. L. Kuhn and Dr M. B. Schaffler for their helpful suggestions. The authors also thank Mr D. Kayner for technical support on testing machine development and the Bone and Mineral Research Laboratory at the Henry Ford Hospital for technical advice on SEM analysis.

\section{REFERENCES}

Ascenzi, A. and Bonucci, E. (1976) Relationship between ultrastructure and 'Pin Test' in osteons. Clin. Orthop. Rel. Res. 121, 275-294.

Baker, J., Frankel, V. H. and Burstein, A. H. (1972) Fatigue fractures: biomechanical considerations. J. Bone Jt Surg. 54A, 1345-1346.

Bargren, J. H. and Tilson, D. H. (1971) Prevention of displaced fatigue fractures of the femur. J. Bone Jt Surg. 53A, 1115.

Burr, D. B., Martin, R. B., Schaffler, M. B. and Radin, E. L. (1985) Bone remodeling in response to in vivo fatigue damage. J. Biomechanics 18, 189-197

Burr, D. B., Schaffler, M. B. and Frederickson, R. G. (1988)
Composition of the cement line and its possible mechanical role as a local interface in human compact bone. J. Biomechanics 21, 939-945.

Burstein, A. H., Currey, J. D., Frankel, V. H. and Reilly, D. T. (1972) The ultimate properties of bone tissue: the effects of yielding. $J$. Biomechanics 5, 35-44.

Carter, D. R. and Caler, W. E. (1985) A cumulative damage model for bone fracture. J. orthop. Res. 3, 84-90.

Carter, D. R., Caler, W. E., Spengler, D. M. and Frankel, V. H. (1981) Uniaxial fatigue of human cortical bone: the influence of tissue physical characteristics. J. Biomechanics 14, 461-470.

Carter, D. R. and Hayes, W. C. (1976) Fatigue life of compact bone-I. Effects of stress amplitude, temperature and density. J. Biomechanics 9, 27-34

Carter, D. R. and Hayes, W. C. (1977) Compact bone fatigue damage--I. Residual strength and stiffness. J. Biomechanics 10, 325-338.

Carter, D. R., Hayes, W. C. and Schurman, D. J. (1976) Fatigue life of compact bone-II. Effects of microstructure and density. J. Biomechanics 13, 27-38.

Choi, K. and Goldstein, S. A (1989) The fatigue properties of human trabecular bone tissue. Proc. 13th Am. Soc. Biomechanics, pp. 24-25. Burlington, Vermont.

Choi, K., Kuhn, J. L., Ciarelli, M. J. and Goldstein, S. A (1990) The elastic moduli of human subchondral, trabecular, and cortical bone tissue and the size-dependency of cortical bone modulus. J. Biomechanics 23, 1103-1113.

Corondan, G. and Haworth, W. L. (1986) A fractographic study of human long bone. J. Biomechanics 19, 207-218.

Currey, J. D. (1962) Stress concentrations in bone. J. Micro Sci. 103, 111-133

Devas, M. B. (1958) Stress fracture of the tibia in athletes or'shin soreness'. J. Bone Jt Surg. 40B, 227.

Evans, F. G. and Lebow, M. (1957) Strength of human compact bone under repetitive loading. J. appl. Physiol. 10, 127-130.

Evans, F. G. and Riolo, M. L. (1970) Relationship between the fatigue life and histology of adult human cortical bone. J. Bone Jt Surg. 52A, 1576-1586.

Freeman, M. A. R., Todd, R D. and Pirie, C. J. (1974) The role of fatigue in the pathogenesis of senile femoral neck fractures. J. Bone $J t$ Surg. 56B, 698-702.

Frost, H. L. (1960) Presence of microscopic cracks in in vivo bone. Henry Ford Hosp. Bull. 3, 25-35.

Frost, H. L. (1966) Bone Dynamics in Osteoporosis and Osteomalacia. Charles C. Thomas, Springfield.

Gray, R. J. and Korbacher, G. K. (1974) Compressive fatigue behavior of bovine compact bone. J. Biomechanics 7 , 287-292.

Griffiths, W. E. G., Swanson, S. A. V. and Freeman, M. A. R. (1971) Experimental fatigue fracture of the human cadaveric femoral neck. J. Bone Jt Surg, 53B, 136.

Hollister, S. J., Fyhrie, D. P., Jepsen, K. J. and Goldstein, S. A (1990) Application of homogenization theory to the study of trabecular bone mechanics. J. Biomechanics (accepted).

Jee, W. S. S. (1983) The skeletal tissue, In Histology: Cell and Tissue Biology (edited by Weiss, L.), pp. 200-255. Elsevier, Amsterdam.

Keller, T. S., Lovin, J. D., Spengler, D. M. and Carter, D. M (1985) Fatigue of immature baboon cortical bone J. Biomechanics 18, 297-304.

Kendall, K. (1975) Control of cracks by interfaces in composites. Proc. R. Soc. Lond. 341, 409-428.

Kragstrup, J. and Melsen, F. (1983) Three-dimensional morphology of trabecular bone osteons reconstructed from serial sections. Metab. Bone Dis. Rel. Res. 5, 127-130.

Kuhn, J. L., Goldstein, S. A., Choi, K., London, M. Feldkemp, L. A. and Matthews, L. S. (1989) A comparison of the trabecular and cortical tissue moduli from human iliac crests. $J$. orthop. Res. 7, 876-884

Lafferty, J. F. (1978) Analytical model of the fatigue characteristics of bone. Aviation Space Environ. Med. 49, 170-174. 
Lafferty, J. F. and Raju, P. V. V. (1979) The influence of stress frequency on the fatigue strength of cortical bone. J. biomed. Engng 101, 112-113.

Martens, M., van Audekercke, R., de Meester, P. and Mulier, J. C. (1986) Mechanical behavior of femoral bones in bending loading. J. Biomechanics 19, 443-454.

Martin, R. B. and Burr, D. B. (1982) A hypothetical mechanism for the stimulation of osteonal bone remodeling by fatigue damage. $J$. Biomechanics 15, 137-139.

Martin, R. B. and Ishida, J. (1989) The relative effects of collagen fiber orientation, porosity, density, and mineralization on bone strength. J. Biomechanics 22, 419-426.

Martin, R. B. and Pramanik, J. (1989) Effects of aspect ratio on the bending behavior of bone and wood. Proc. $13 \mathrm{th} \mathrm{Am}$. Soc. Biomechanics, pp. 22-23. Burlington, Vermont.

McBryde, A. M. (1976) Stress fractures in athletes. J. Sports Med. 3, 212.

Moyle, D. D. and Bowden, R. W. (1984) Fracture of human femoral bone. J. Biomechanics 17, 203-213.

Moyle, D. D., Welborn, J. W. and Cooke, F. W. (1978) Work to fracture of canine femoral bone. J. Biomechanics 11, 435-440.

Parfitt, A. M. (1983) The physiologic and clinical significance of bone histomorphometric data. In Bone Histomorphometry: Techniques and Interpretation (Edited by Recke, R. R.). CRC Press, Boca Raton, Fl.

Pattin, C. A., Carter, D. R. and Caler, W. E. (1990) Cortical bone modulus reduction in tensile and compressive fatigue. Trans. 36th Orthop. Res. Soc. 15, 50.

Pedersen, D. R., Brown, T. D. and Brand, R. A. (1990) Bone ingrowth stress distributions within a novel prosthesis anchorage layer. Trans. 36th Orthop. Res. Soc. 15, 205.

Radin, E. L. (1972) Trabecular microfractures in response to stress: The possible mechanism of Wolff's law. Proc. 12th Congress Internat. Soc. Orthop. Surg. and Trauma, pp. 59-63. Tel Aviv.

Reilly, D. T. and Burstein, A. H. (1974) The mechanical properties of cortical bone. J. Bone $J$ t Surg. 56A, $1001-1022$

Rice, J. C., Cowin, S. C., and Bowman, J. A. (1988) On the dependence of the elasticity and strength of cancellous bone on apparent density. J. Biomechanics 21, 155-168.

Schaffler, M. B. and Burr, D. B. (1988) Morphology of the osteonal cement line in human bone. Anat. Rec. 217, 223-228.

Schaffler, M. B. and Burr, D. B. and Fredrickson, R. G. (1987) Stiffness of compact bone: the effects of porosity and density J. Biomechanics 21, 13-16.

Schaffler, M. B., Radin, E. L. and Burr, D. B. (1989) Mechanical and morphological effects of strain rate on fatigue of compact bone. Bone 10, 207-214.

Swanson, S. A. V., Freeman, M. A. R. and Day, W. H. (1971) The fatigue properties of human cortical bone. Med. Biol. Engng 9, 23-32.

Tarnopol'skii. IU. M. and Kinics, T. (1985) Static Test Methods for Composites, pp. 221-252. Van Nostrand, New York.

Torzilli, P. A.. Takebe, K., Burstein, A. H. and Heiple. K. G. (1981) Structural properties of immature canine bone. J. biomech. Engng 103, 232-238.

Watson, M. (1975) Microfractures in the head of the femur. J. Bone jt Surg. 57A, 696.

Wright, T. M. and Hayes, W. C. (1976) Tensile testing of bone over a wide range of strain rates: effect of strain rate, microstructure and density. Med. Biol. Engng 14, 671-679. 